\title{
CURVE FITTING AND THE TRANSMISSION INTEGRAL:
}

WARNINGS AND SUGGESTIONS

by

G. K. Shenoy, J. M. Friedt, H. Maletta, and S. L. Ruby

This report was prepared as an itecoutit of work sponsored by the Lnited States fiuverrment. Veither the United States nor the United States Atumic Fnergy Cominission, nor any of their employees. nur any of Cornmission, hor any of their emplones. nur any or

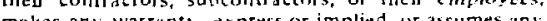

makes any warranty. express or impled. or assumes any

lepal liahility or responsibality for the accuracy, com -

pleceness or usefulness of ary information, apraratus.

product or piocess disclosed, ir represents
would not infringe privately owned rithts.

\section{Presented at:}

Ninth Symposium on Mössbauer Effect Methodology, Chicago, Illinois, February 1974 
Ninth Symposium on Mössbauer Effect Methodology, Chicago, Februa ry 1974

CURVE FITTING AND THE TRANSMISSION INTEGRAL: WARNINGS AND SUGGESTIONS

G. K. Shenoy and J. M. Friedt

Laboratoire de Chimie Nucléaire Centre de Recherches Nuclëaires 67200 strasbourg, France

H. Maletta

Institut für Festkorperforschung der Kernforschungsanlage, 517 Jülich 1. Federal Republic of Germany

S. I. Ruby ${ }^{*}$

Argonne National Laboratory

Argonne, Illinois 60439 


\section{INTRODUCTION}

Every spectroscopy has developed its own techniques of spectral. analysis and Móssbauer spectroscopy is no exception to the rule. The earliest work on the detailed spectral shape of nuclear resonance gamma rays date back to the papers of Margulies and Ehrman and Ruby and Hicks.? In these they showed the importance of absorber and source thicknesses on the shape of individual absorption lines. However, most of us in the last 12 years have been satisfied to describe them in their simplest approximation, i.e. by a sum of Lorentzian lines whose amplitudes, widths, and positions are determined by the parameters of the hyperfine interactions. This 'thin" approximation neglects the effects due to saturation by a thick absorber. Occasionally, the inaccuracies resulti.ng from the use of the simple approximation in the analysis of partially resolved spectra have been demonstrated; but any enthusiasm for using the transmission integral, described in the above mentioned references, was curbed by the necessity for more elaborate computational facilities.

There have been numerous attempts to overcome this drawback, mainly by expressing the absorption proilie in terms of a fast converging mathematical series. Most of this work has been done by Heberle, Cleveland and their collaborators. 3,4 It was centered around the problems of iron-ramely of one, two or six lines. The one line and two line problems have been dealt with by numerous authors who presented certain correction schemes to the "thin" analysis to account for the absorber thickness effects. In situations involving more than two overlapping lines, or where the absorption shape is not really based on Lorentzian shapes at all (e.g. relaxat: on spectra), and where the absorber is reasonably thick (as is usually desired to get adequate dica), no convenient, fast procedure has been generally available. More precisely, transmission integral programs have generally not been fast enough to run as part of least-mean-square minimization techniques.

During the last few years, the pressure of more difficult measurements and need for better results has pushed several groups toward improved procedures. The work of the group centered at ORNL is especially to be noticed. 5,6,7 Another way to approach the problem was introduced into the literature 
by the group at Hamburg. 8 They use an accurate (albiet slow) convolution of the expected transmission with the flux to produce a properly calculated Mössbauer spectrum. This is then given to a minimization routine using the 'thin' approximation to produce "fitted" values for the parameters. By comparing the "fitted" with the input values of the transmission parameters, one is made aware of the possible problems, and even given information for making plausible corrections. We call such graphs 'awareness' diagrams, and will display some below.

Another technique suggested was introduced at the 7 th Methodology Symposium by Ure and Flinn. Their procedure is to deconvolute the experimental data using the known properties of the flux and fast Fourier transform algorithms; the result is (nominally) the absorber's transmission. After taking its logarithm, the "thin" approximation is no longer approximate, and the minimization can proceed unhindered. Their article, and the following talk by Lin and Preston can be consulted for further information on the convenience and accuracy of this procedure.

Recently, Ted Cranshaw 10 of Harwell has made a major step toward a fast transmission integral routine by pointing out that the flux and transmission need be calculated only once, and that the counting rate at any velocity is found by convoluting these fixed results with an appropriate velocity offset. We have utilized his idea twice-once in a straightforward way, and second, by combining it with Gauss-Legendre integration techniques, looking toward even faster programs.

The purpose of our talk today is to warn you of the dangers in the "thin" approach for the analysis of the partially resolved spectra. We shall present some awareness diagrams described above for a variety of physical and chemical problems. In addition we will discuss the analysis of some actual data using the above-mentioned convolution procedures. For those of you with access to substantial computational facilities, we offer the Gauss-Legendre as well as Cranshaw algorithms as Fortran IV programs. 


\section{THE TRANSMISSION INTTEGRAL}

We wish to compute the counting rate in the detector as a function of the relative velocity of source and absorber. It is convenient to describe the flux distribution from the source in three parts:

$$
\begin{aligned}
& \text { FLUX }(E, v)=B+S\left(1-f_{S}\right)+\operatorname{Sf}_{S} F(E, v) \\
& F(E, v)=\left(\frac{2}{\pi \Gamma_{S}}\right)\left(1+\left(\frac{E-E_{o}-E(v / c)}{\Gamma_{s / 2}}\right)^{2}\right)^{-1}
\end{aligned}
$$

where $B$ is the background counting rate, $S$ is the integrated signal rate, $f_{f}$ is the source resonant fraction, $E$ is the nominal $\gamma$-ray energy, and $E$ is the actual energy。 The transmission ot the first two terms is practically independent of $\mathrm{E}$ and $v_{\text {. }}$. For the last term the transmission is

$$
\operatorname{TRANSS}(E)=\exp \left[-T_{a}^{*} A(E)\right]
$$

where $T_{1}=n \sigma_{f}$ is the absorber thickness (since $n$ is the number of resonant nuclei per $\mathrm{cm}^{2}$, and $\sigma_{o}$ is the cross section per nucleus, $f_{a}$ is the absorber resonant fraction, $T_{a}$ is dimensionless) and $A(E)$ is the normalized absorption shape, to be discussed below. The transmitted flux then becomes

$$
\begin{aligned}
\operatorname{Rate}(v) & =\int F \operatorname{LUX}(E, v) * \operatorname{RANS}(E) d E \\
& =B+S\left(1-f_{s}\right)+S f_{s} \int F(E, v) * T \text { RANS }(E) d E \\
& =R_{0}\left(1-f_{s} \frac{S}{S+B}(1-T I(v)) .\right.
\end{aligned}
$$

Note the pertinent properties of the source and detector are all summarized in the number

$$
F S B=f_{s} \frac{S}{S+B}
$$

while the absorber is fully described by its thickness $T_{A}$ and the normalized absorption shape $A(E)$. The name TI abbreviates 'transmission integral.' 
The absorption shape $A(E)$ is determined in standard fashion from the spins of the excited and ground nuclear states, from the hyperfine interaction parameters, the nuclear moments, etc. The only linfamiliar variation is the normalization required in order for $T_{A}$ to have its conventional meaning. For the simplest case of a single Lorentzian absorber line, A becomes

$$
\mathrm{A}(\mathrm{E})=\frac{\Gamma_{0} / \Gamma_{a}}{1+\left(\frac{\mathrm{E}-\mathrm{E}_{1}}{\Gamma_{\mathrm{a}} / 2}\right)} 2=\frac{\Gamma_{0} / \Gamma_{a}}{1+\mathrm{x}^{2}} .
$$

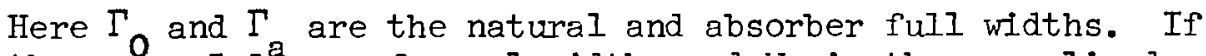
there are $I$ lines of equal width, and $W_{i}$ is the normalized Clebsch-Gordan intensity for the ith line, A becomes:

$$
A(E)=\Gamma_{o} / \Gamma_{a} \sum_{i=1}^{L} \frac{w_{i}}{1+\left(x-x_{i}\right)^{2}} .
$$

More generally, the absorber shapes are not limited to sums of Lorentzians. Relaxation spectra in particular give rise to more exotic shapes. The important rule for normalizing $A$ is that its integral must always have the same value, namely

$$
\int_{-\infty}^{\infty} A(E) d E=\Gamma_{0} \pi / 2
$$

It should be noted that FSB presents a minor problein. If it is known from auxiliary experiments, then it should be used, and $\mathrm{T}_{\mathrm{a}}$ will have the desired absolute significance. If unknown, there is a temptation to make it a variable of the fit. In some cases with very thick absorbers, this may be appropriate, but usually good fits will be difficult to obtain. Roughly, FSB and $\mathrm{T}_{\mathrm{a}}$ are 'parallel' variables; increasing either stretches the vertical scale in a very similar way, and the computer naturally has trouble separating the two. It is usually better to fix FSB with an approximate value.

In the next section we describe two TI procedures that we have used. 


\section{CONVOLUTION PROCEDURES}

\section{3a. Gauss-Legendre Quadrature Technique}

The principal effor in obtaining the exact spectral shape is of performing the numerical integration in $\mathrm{Eq} .(3)$ to an accuracy sufficient to describe the experimental situation. This can easily be done by the Gauss-Legendre quadrature technique, 11 which is applied here to every zone into which the region of interaction is divided. The integrand in Eq. ( 1 ) is firstly divided into two major regions, the inner region and the wings. The inner region is bounded approximately by the usual velocities employed in the experiments, and is subdivided into smaller zones by laying a uniform grid. In the wings, the size of the zones increases with a power law, and the limits of the integration is thus extended up to about $10^{5}$ to $10^{6}$ times the line width. The integral is now evaluated for each zone, using 12 integration steps. The numiber of grids in the inner region itself varies from 2 to 20 depending on the experimental velocity range and the source and absorber line widths.

Note tinat the transmission is calculated only once per spectrum, a la Cranshaw. But flux must here be recomputed anew for every experimental velocity. However this program is rather easily adapted to accommodate experiments performed with non-linear drives. In Appendix A we give a complete FORTRAN listing of a program that one can use. The writeup is such that it can easily be adapted to various physical situations. 12,13

In the program given, the number of integration steps is set equal to 12 . The inner region is divided into subzones of width equal to the sum of source and absorber linewidths. For test purposes, the number of these subzones has been increased or decreased by the parameter NADSUB. The procedure described here, with variations, has been successfully used in a variety of problems.

\section{3b. The Cranshaw Technique}

The main point seen by Cranshaw is that FLUX and TRANS in Eq. (3) need only be calculated once. To produce all the different rates at every velocity, it is always the same flux shape and the same transmission that needs to be convoluted- the only change is their relative displacement. 
In fact, if we trink of FIUX and TRANS as arrays, FLUX(N,M) and TRANS(N) where $N$ and $M$ are index numbers, then

$$
\text { Rate }(M)=\sum_{N} F L U X(N, M) * T \text { RANS(N). }
$$

Once FIUXX and TRANS have been computed, then each rate at a new velocity is only some 400 multiplications and sums. This must be repeated for each point in the spectrum.

There are, of course, many details in achieving this result and we have attempted to write the program in a way which makes it quite easy to vary from one problem to another (See Appendix B). The progran has 5 major divisions. The first is just common blocks and dimension statements, and will need to be modified in a straightforward way in changing from one isotope, or Hamiltonian, to another. The second division, called initialization, is mainly concerned with getting the latest values of the interaction parameters ready to go; it will also need changes. The third section, called transmission, uses the values of the parameters to calculate the transmission at every energy. It, too, must be modified for every new problem. The modifications, til.1 now, are no more or less than is needed using the 'thin' approximation. The fourth section, which calculates flux, will rarely be modified, since we nearly always use a simple Iorentzian shape here. The last and fifth section, called convolution, should never need changing from one problem to another. One exception is where the desired spectrum has a center of symmetry. It is then faster to calculate up to this center, and get the remainder by folding and interpolation. This has been left out here for simplicity.

An important program choice is called Covvo. For CONVO $=0$, then the program does not proceed beyond the third section, produces the 'thin' approximation, and behaves like the rapid routines we had been used to. For CONVO $=1$, the program does the complete transmission integral properly. Initial fits are made with convo $=0$ uintil one understands the problem rather well; finally, fits with CONVO $=1$ will either give better fits or show that the 'thin' approximation was adequate.

\section{SOME APPLICATIONS OF THE TRANSMISSION INTEGRAL}

4a. Hyperfine Magnetic Interactions

The error introduced in the determinations of either the ratio of the nuclear $g$-factor of the levels in a 
Mössbauer transition or the magnetic field at the nucleus through the 'thin' analysis of a hyperfine magnetic spectrum has been discussed at various places.14,15,16

In Fig. 1 we reproduce the 'awareness' diagram given by Gerdau et al. 8 for the case of $2 \rightarrow 0$ tiansition and $a$ magnetic interaction. A large number of rotational states among the rare earth and the actinide nuclei are analogous cases. We notice from Fig. I that the error would be some $17 \%$ in the determination of the excited state splitting when the splitting is equal to the onservable line width for an absorber thickness equal to 10.

A particularly illustrative example on the accurate determination of the ratio of the nuclear $g$-factor is given by Gerdau et al. for the $81-\mathrm{keV}, \frac{5}{2} \rightarrow \frac{1}{2}$ transition in $133 \mathrm{Cs}$. in Fig. 2 we give the experimental data with least-squares fit obtained using both a 'thin' and TI analysis. In Fig. 3 the vailues of $x^{2}$, the goodness-of-fit parameter, has been plotted as a function of the ratio of the nuclear g-iactors. It is interesting to observe that $\gamma^{2}$ goes through a sharp minimum for a jarticular value of Eex/ggd only when the transmission integral is employed in the spectral analysis.

The binary alloys of the $3 d$ elements have been investigated over many years to try to understand the relative contributions to the inf magnetic field from various causes. 17 Often the Mössbauer results have been found to deviate from corresponding spin-echo measurements. 18 One of the likely reasons 19 for such discrepancies could be the absorberthickness effect. Another experimental area where absorber saturation effects are likely to be of importance is in the study of the critical region in ordered nagnets. As one raises the temperature, the resonance lines approach ever closer to each other and the overall absorption shape becomes much deeper, narrower, and unresolved. Even if the 'thin' approximation were adequate well below $\mathrm{T}_{\mathrm{c}}$, it is unlikely to remain so when near $\mathrm{T}_{\mathrm{c}}$. 


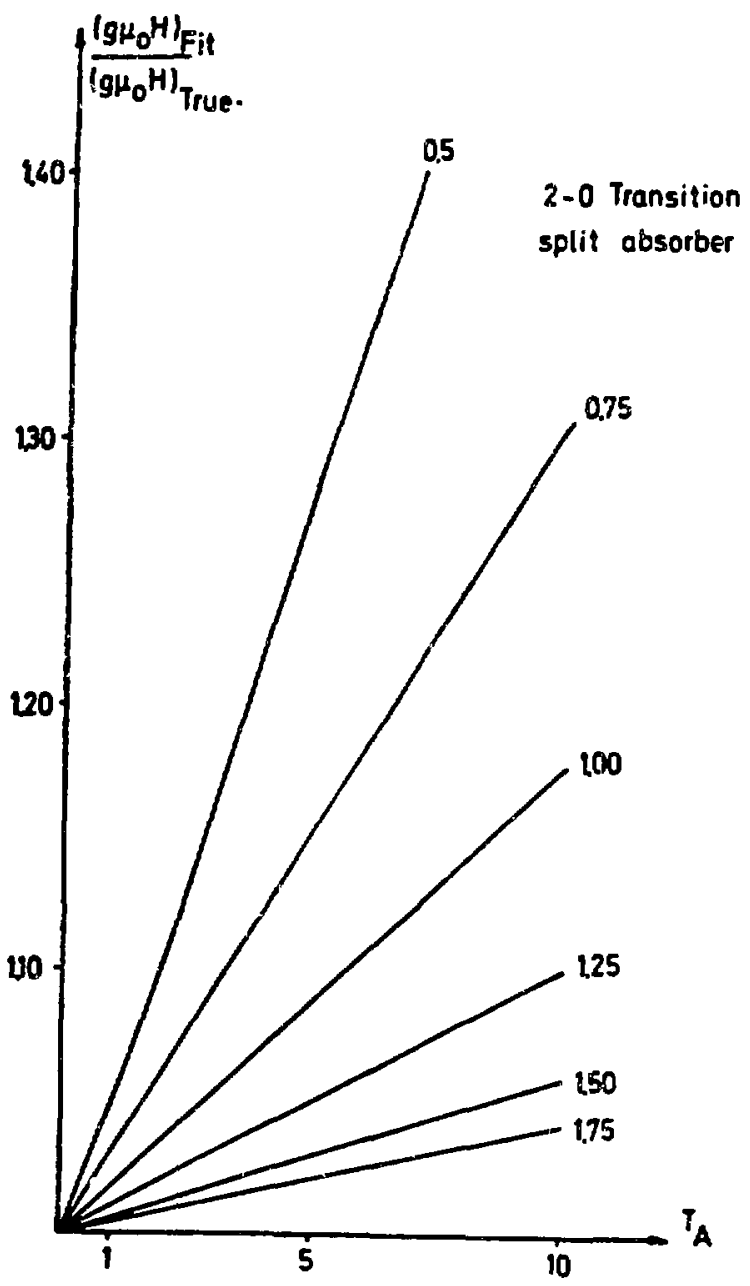

Fig. 1. Awareness diagram for the magnetic hyperfine interaction in the $2 \rightarrow 0$ transitions. The number on each line gives the value of $\left(g \mu_{0} \mathrm{H} / \Gamma_{0}\right)_{T R U E}$
Ref. 8 ] 

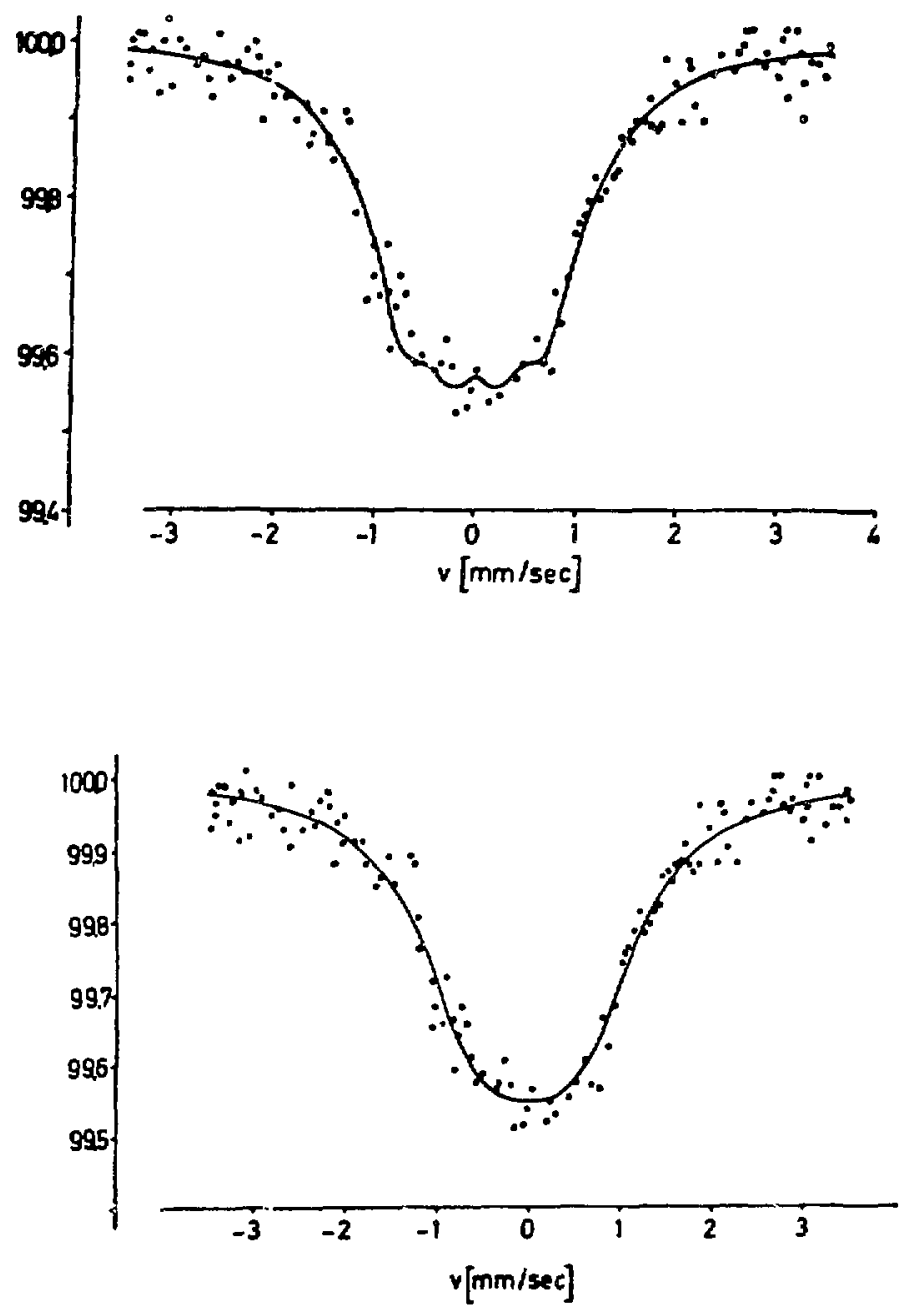

Fig. 2. The spectrum of $133 \mathrm{CsCl}$ in $65 \mathrm{kOe}$ external field. The solid curves show results of 'thin' (below' $j$ and transmission integral (above) analysis [Ref. 8]. 



Fig. 3. The value of $\chi^{2}$ as a function of $g_{e x} / g_{g d}$ for $133 \mathrm{Cs}$ data shown in Fig. $3 \mathrm{~b}$ and $3 a$ from 'thin' and transmission integral analysis [Ref. 10].

4b. Quadrupole Patterns

Most of the remarks made in Sec. $4 a$ are also applicable to the analysis of quadrupole patterns. The awareness diagrams for the Móssbauer transitjons involving states with $\frac{3}{2}$ and $\frac{1}{2}$ have been discussed by various authors.8,20 In Fig. 4 we give again the awareness diagram for this case.

For the Mössbauer transitions involving higher spins usually the quadrupole spectra are only partially resolved. The quadrupole spectra of the $37-\mathrm{keV}\left(\frac{3}{2} \rightarrow \frac{5}{2}\right)$ resonance in ${ }^{12} \mathrm{ISb}_{\mathrm{Sb}}$ is a typical example of such a situation. Errors as 
large as $60 \%$ are possible in certain circumstances as shown in Fig. 5.13 The deduction of the asymmetry parameters $\eta$ for such cases is even more difficult. In another work ${ }^{12}$ we have compared the values of $\mathrm{eQV}_{\mathrm{zz}}$ in some $\mathrm{Sb}$ corpounds obtained from the Mossbauer spectra analyzed with the 'thin' approximation with those obtalned from NQR spectroscopy. These deviations essentially disappeored when the data were analyzed with the transmission integral.

The case of Mössbauer transitions involving other spins will not be dealt with here, but awareness diagrams have been published for such measurements. 8,15

\section{4c. Isomer Shifts}

We remark that the errors in isomer shift determinations caused by 'thin' analysis will be small even for quite thick samples if the spectra themselves are symetric. Such situations are realized, for example, in pure magnetic hyperfine spectra. The thin analysis will, however, not be sufficient to yield accurate isomer shift from asymmetric hyperfine patterns, which usually involve quadrupole interaction. The effects are especially serious in the case of $2 \rightarrow 0$ interband transitions of the rare earth, $5 \mathrm{~d}$, and the actinide nuclei. The isomer shift in these cases is usually small and many of their compounds give unresolved quadrupole patterns. An awareness diagram for this case has been reported by Hershkowitz et al. 15 . In Fig. 5 , we have illustrated the case of the transition involving $\frac{7}{2}-\frac{5}{2}$ spins.

\section{4d. Gol'danski 'i-Karyagin Effects}

The Gol 'danski 'i-Karyagin effect ${ }^{2 I}$ (GKF) has now been reported as observed in the quadrupole spectra of a large number of nuclei. It resulis from the fact that $\left\langle x^{2}\right\rangle$ for an atom is generally not the same in all crystal directions; correspondingly, the Debye-Waller resonant fraction must vary in different crystal directions. Since the hyperfine fields are locked to the sarne axes, this means that the single-crystal line intensities are disturbed from the simple predictions, and even after the crystals are powdered and randomized in direction, the relative line intensities deviate from the normali zed Clebseh-Gordan coefficients $W_{i}$. If the above effect happened to attenuate the stronger lines 




Fig. 4. Awareness diagram for the symmetric quadrupole hyperfine interaction in $\frac{3}{2} \rightarrow \frac{1}{2}$ transitions. The number on each curre gives $\left(\mathrm{eQV}_{\mathrm{zZ}} / \Gamma_{\mathrm{O}}\right)_{\mathrm{TRUE}}[\operatorname{Ref} .8]$. 


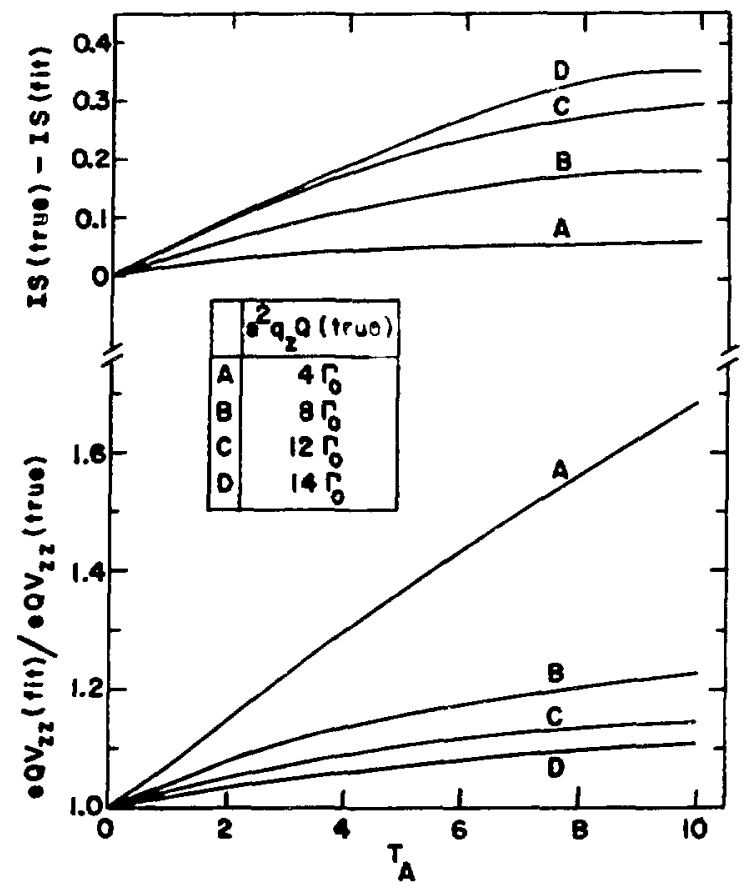

Fig. 5. Awareness diagrams for $\frac{7}{2} \rightarrow \frac{5}{2}$ quadrupole ${ }^{12 I_{S b}}$ spectrum. Errors for both the quadrupoleinteraction and isomer-shift parameters are shown. Unit for isomer shifts is millimeter/sec.

more than the weaker ones, the observed effect would look rather like that resulting from attenuation by a thick absorber.

We demonstrate these effects, in spectra from ${ }^{12} I_{S b}$ in an axial field gradient. This is a $\frac{7}{2}-\frac{5}{2}$ transition. Curve a of Fig. 6 shows the quadrupole spectrum, including GKF, 12 as calculated in the thin approximation. Curve $b$ of this figure is the same without GKE, but for varying absorber thicknesses while using the proper transmission integral. In comparison, indeed the GKE and absorber saturation have similar effects on the spectrum.

An experimentaliy measured spectrum (even with $\mathrm{T}_{\mathrm{a}}<1$ ) can be mistaken as evidence for the presence of GKE with 


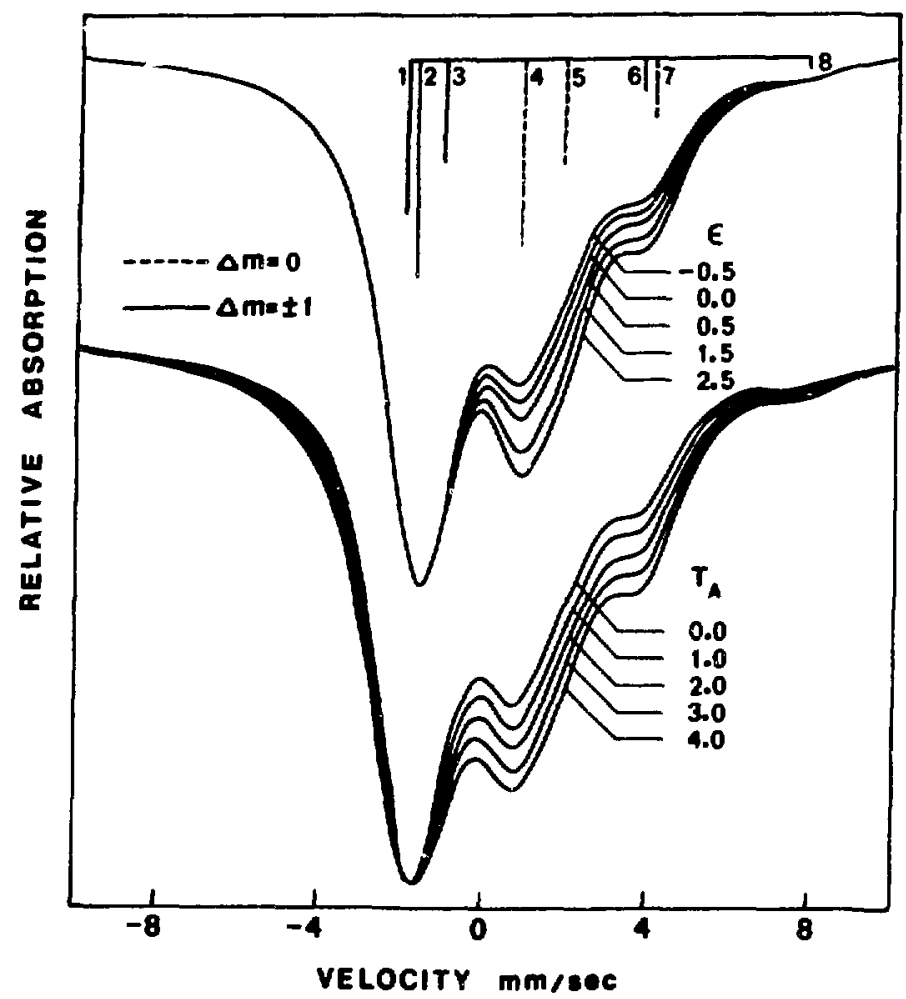

Fig. 6. a) Quadrupole spectra for ${ }^{12 l_{S b}}$ with $\mathrm{eQV}_{\mathrm{zz}}=-20 \mathrm{~mm} / \mathrm{sec}, \Gamma_{\mathrm{O}}=1.05 \mathrm{~mm} / \mathrm{sec}, \eta=0$, and for various values of $\epsilon=\mathrm{k}^{2}\left(\left\langle\mathrm{z}^{2}\right\rangle-\left\langle\mathrm{x}^{2}\right\rangle\right)$ calculated in the thin approximation. b) The transmission integral simulated spectra for above case with $\epsilon=0$, but for different absorber thicknesses. All spectra are normalized to the same maximum dip。

$\left.\epsilon=\left\langle\left\langle\mathrm{z}^{2}\right\rangle-\left\langle\mathrm{x}^{2}\right\rangle\right) \mathrm{k}^{2}\right\rangle 0$. Here $\left\langle\mathrm{z}^{2}\right\rangle$ and $\left\langle\mathrm{x}^{2}\right\rangle$ are the meansquare displacement parallel and perpendicular to the principal symmetry axis, and $k$ is the absolute value of the wave vector for the $\gamma$ radiation. 


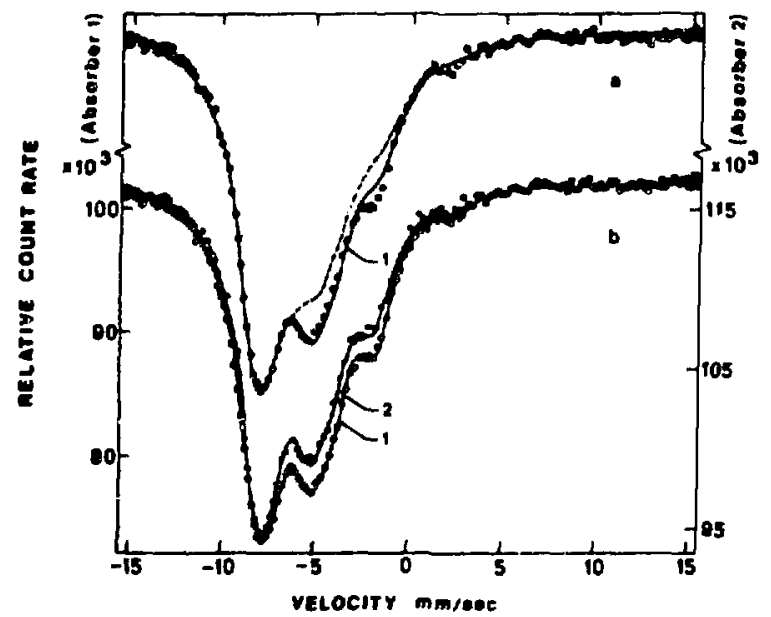

Fig. 7. Mössbauer quadrupole spectra of $\mathrm{ib}\left(\mathrm{CH}_{3}\right)_{3} \mathrm{Br}_{2}$ against a $\mathrm{Ba} 12 . \mathrm{mSnO}_{3}$ source, bnth at $4 . ? \mathrm{~K}$ ? for two absorber thîcknesses: (1) $17 \mathrm{mp} / \mathrm{cm}^{2}$ and (2) $7 \mathrm{mg} / \mathrm{cm}^{2}$ of $\mathrm{sb}$. In (a) the solid curve represents the thin approximation including the GKE; the dashed curve, without the GKE. In (b) the data for the two absorbers are fitted by TI without any GKE.

The spectra of $\mathrm{Sb}\left(\mathrm{CH}_{3}\right)_{3} \mathrm{Br} 2$ measured for two absorber thicknesses against a $\mathrm{BaIZImSnO}_{3}$ source, both at $4.2 \mathrm{~K}$ is presented in Fig. 7. In this compound the site symmetry of $\mathrm{Sb}$ ions is $\mathrm{D}_{3 \mathrm{~h}}$. The dashed curve (a) in Fig. 7 is the best fit obtained from a 'thin' analysis assuming $\varepsilon=0$. Inclusion of GKE in this analysis considerably improves the fit (solid curve (a) in Fig. 7) except in the region of line 7 (see Fig. 6). Such an analysis with two absorber thicknesses, however, did not yield identical values for $\epsilon$. In Fig. 7 , curves (b), we give the fits for two absorbers using the transmission integral $\left(E q_{0}(3)\right)$ without including any GKE. These fits are more satisfactory than those of curves (a) on several grounds. Thus the transmission integra? analysic has removed the evidence for the presence of nonzero GKE parameters in this compound. 
The discussion given above is not unique to ${ }^{12 l_{S b}}$ spectra and is likely to be realized in spectra involving different Móssbauer transitions.

Single-crystal absorbers, of course, create spectra for which the relative line intensities are different from those of powdered absorbers. If a single crystal absorber is made thick, a new effect makes its appearance. The source $\gamma$ rays are actually not all of one type, but can be described as a mixture of two polarizations, say parallel and perpendicular to the principal axts of the absorber. These can have different absorption cross sections, and the transmission now must be treatea as referring to the sum of the transmissions of the two polarizations. We will not treat this problem here, and refer the interested reader to the useful and clear work of Housley et al., 22 and of Pfannes and Gonser. 23

\section{He. Interferenes iffects}

There are two ways to produce a final state consisting of the de-excited source nucleus and an absorber atom with $a$ hole in its $\mathrm{k}$ shell. One is nuclear-resonance absorption followed by $\mathrm{k}$-shell internal conver'sion; another is the ordinary electronic-photoelectric effect. The amplitudes of these processes must be added, and when the sum is squared to find the liklihood of such processes, a cross term can result. It is called a dispersion term for it modifies the normal Lorentzian shape similarly to dispersion in resonant absorption of ordinary light. In fact, Eq. (4) is changed to

$$
A(E)=\Gamma_{a} \frac{1-2 \xi x}{1+x^{2}},
$$

where $\xi$ is the amplitude of the dispersion or interference term.

Such effects have been observed in EI, E2 and mixed E2/MI transitions.?,24,25,26 It has been observed that the dispersion effect seems to be enhanced by using thick absorbers. 25

Erickson et al. 7 have made such analyses in connection with their study of the dispersion term in $197 \mathrm{Au}$. The 


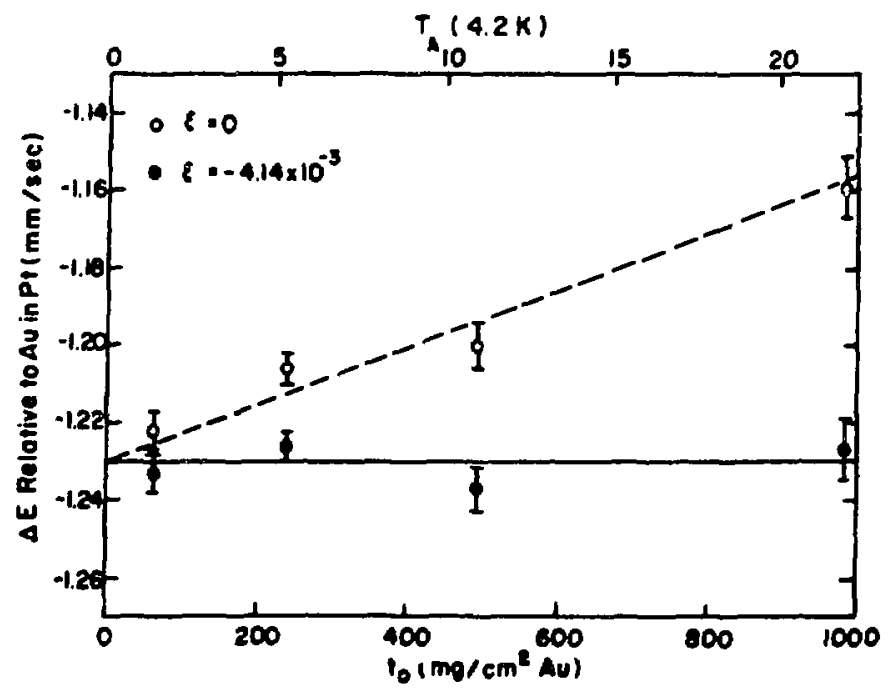

Fig. 8. Isomer shift $\triangle E$ versus Au-metal absorber thickness. The dashed curve is 'thin' with the interference parameter $\xi=0_{\xi}$ while the solid curve uses $\xi=-4.14 \times 10^{-3}$ and $\mathrm{TI}$ analysis $[\operatorname{Ref} 7$ ].

deduced isomer shifts will be subject to error if either the dispersion term or absorber thickness effects are neglected. In Fig. 8, Erickson's isomer shift data for various thickness with and without the dispersion term are given. Small isomer shifts can be readily 'created.' These effects will be of importance for the measurement of the small isomer shifts in the $2 \rightarrow 0$ transitions; here E2 transitions tend to create relatively large values of $\xi$.

\section{4f. Mössbauer Thermometer}

The basis of the Mössbauer low-temperature thermometer has been discussed by Kalvius et al. 27 The temperature is arrived from the relative intensities of the various hyperfine lines as these are affected by the temperature-dependent Boltzmann populitions of the ground stace hyperfine levels of the resonant absorber nuclei. Among the various 
Mössbauer transitions suggested for the absorber thermometer, the $21.6-\mathrm{keV}$ transition in $15 \mathrm{I}_{\mathrm{Eu}}$ has acceptable characteristics for the temperature range from 25 to $250 \mathrm{mk}$.

A primary difficulty in the use of Mossbauer thermometers has arisen from the fact that the transmitted intensity is not linearly dependent on the absorber thickness, but in fact, requires use of the transmission integral. The absorber thickness for a particular transition in the hyperfine spectrum at low temperatures must be modified from $\mathrm{T}_{\mathrm{A}} \mathrm{W}_{\mathrm{i}}$ (see Eq. (2)) by the factor $\mathrm{P}(\mathrm{m})$ where

where

$$
P(m)=\frac{\exp (-m E / k T)}{\sum_{m=-I g}^{I g} \exp (-m E / k T)}
$$

$$
\mathrm{E}=\mathrm{g} \mu_{\mathrm{n}} \mathrm{I}_{\mathrm{n}}
$$

Here $H_{n}$ is the nuclear magnetic fileld, $g$ is the nuclear ground $^{n}$ state $g$-factor, $\mathrm{kT}$ is the temperature in energy units, and $m$ is the magnetic quantum number for the ground state of this particular nuclear transition.

In order to demonstrate the influence of absorber thickness we show first, in Fig. 9a, spectra for EuS at 4200, 40,60 , and 80 millidegrees as calculated 'thin'; second, in Fig. $9 b$, spectra at 50 millidegrees for $T_{A}=1,4$, and 6 calculated with a transmission integral. Comparison of the two indicates that the 'thin' approximation leads to overestimates of the temperature. Further, for $T_{A}=10(a$ reasonable thickness for an absorber split into five major absorption lines), the apparent temperature is a full $50 \%$ larger than the actual. 28

\section{CONCLUSTONS}

We have attermted to show some of the kinds of errors that can be caused by use of the 'thin' approximation, rather than the transmission integral, in the analysis of Mössbauer data. The so-called 'awareness' plots can give one a feeling for the possible size of the er'ror, if you have a physical case close to one already analyzed. 

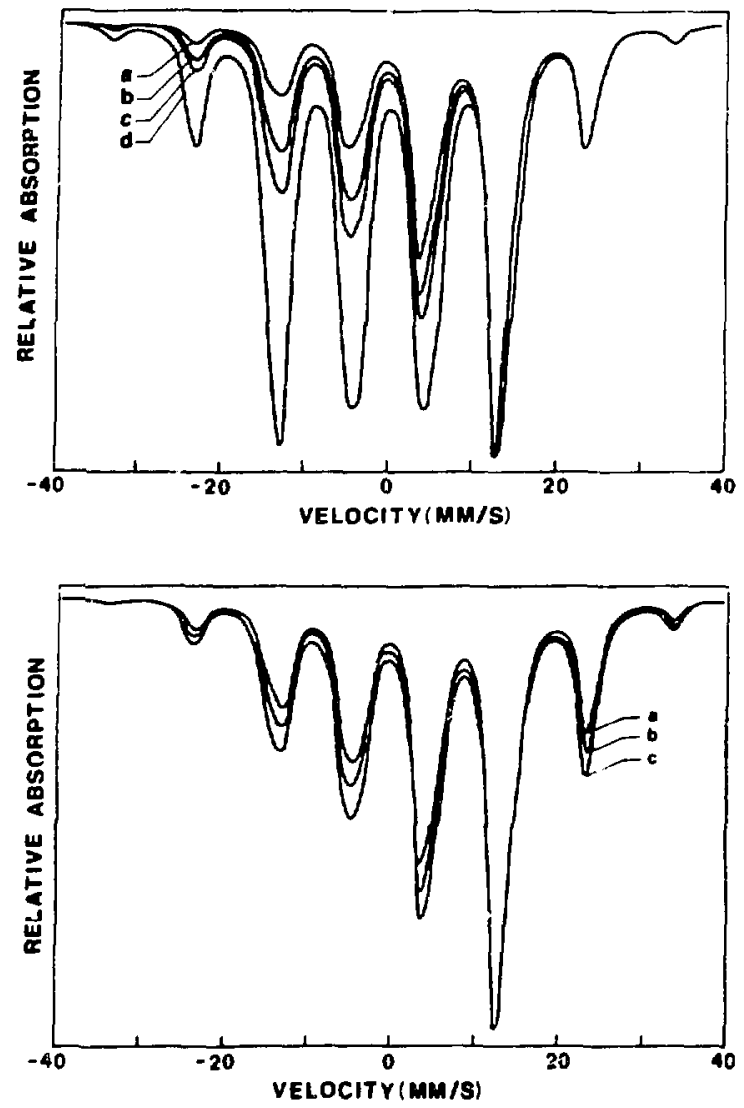

Fig. 9 (a) The hyperfine magnetic spectrum of

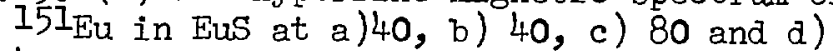
$4200 \mathrm{mk}$ calculated in the thin approximation. (b) The spectrum for EuS at $50 \mathrm{mk}$ and for absorber thickness, $n_{A} \sigma_{O} f_{A}$, of a) 1, b) 4 , and c) $6[\operatorname{Ref} .28]$.

There will still be a majority of cases where the improved technique is not really needed. For example, all well-resolved spectra can be treated as a collection of single lines, and thickness effects can be taken into account by oläer methods. Even with poorly resolved spectra, saturation effects cannot be important if $T_{A} \leqslant I_{0}$ And this condition is enforced in many experiments by such factors as low isotopic enrichment, large absorber dilution by other 
elements, high photoelectric absorption, etc. It is, of course, clear that a small observed effect does not guarantee that $T_{A}$ is small, as this is most often due to a sme.11 resonant fraction in the source, or a poor signal to back-ground ratio in the detector. Other special cases occur where neglect of the transmission integral can de justified; for example, the isomeric shifts in symetrical. spectra will not be affected, even though simul.taneously the magnetic splitting $g \mu_{n} \mathrm{H}$ is being ill treated. Our procedure now is to make most of the initial fits with the transmission integral switched off with CONrO $=0$. After the fits are becoming sensible, then CoNvo $=$ i switches on the slewer transmission integral calculation. A comparison of the final values of the parameters indicates whether it is necessary to continue with the more expensive procedure.

\section{REFERENCES}

1. S. Margulies and $J$. Ro Mhrmar, Nucl." Instr. Methods 12, 131 (1961); S. Margulies, P. Debrunner and $H_{\text {. }}$ Frauenfelder, Nucl. Instr. Methods ?I, 217 (1963).

2. S. L. Ruby and J. M. Hicks, Rev. Sci. Instr. B3, 27 (1962).

3. J. Heberle and S. Franco, Z. Naturforch. 23 a, 1439 (1968).

4. B. T. Cleveland, 7. Naturforsch。27 a, 370 (1972); B. T. Cleveland and J. Heberle, Phys. Lett. 36A, 33 (197I); B.T. Cleveland and J. Heberle, Phys. Lett. 40A, 13 (1972).

5. J. C. Love, F. E. Obenshain, and G. Czjzek, Phys. Rev. 3, 2827 (1971).

6. D. J. Erickson, I. D. Roberts, J. W. Burton, and Jo Oo Thomson, Phys. Rev。 B I, 2180 (1971).

7. D. J. Erickson, J. F。 Prince, I. D. Roberts, Phys。 Rev。 C 8, 1916 (1973).

8. E. Gerdau, W. Räth and H. Winkler, Z. Pnysik 257, 29 (1972).

9. M.C.D. Ure and P.S. Flinn, Mössbauer Effect Methodology, Vol. 7, ed. I. J. Gruverman (Plenum Press, New York, 1971) p. 245 . 
10. T. E. Cranshaw, J. Fhys, F I, I (1974)。

11. See for exampile, To Lerras Methodes nt techniques de I'analyse numerique (Dunod, Parjs, 1.371).

12. G. K. Shenoy and J. M. Frinolt, Phys. Rev. Lett. 3l, 419 (1373).

13. G。 K. Shenoy and J. Mo Friedt, Nucl. Instr。 Methods (in press)。

14. R. E. Meads, B.M. Place, F。 W。 D. Woodhams, and R. C. Clark, Nucl. Instr. Methods 98, 29 (1972).

15. N. Hershkowitz, R. D. Ruth, S. A. Wender, and A. B. Carpenter, Nucl. Instr. Methods 102, 205 (1372).

I6. G. Hembree and D. C. Price, Nucl. Instr. Methods 108, 99 (1973).

17. G. K. Wertheim, Vo Jaccarino, L. R. Walker, and D. W. E. Buchanan, Phys. Rev. Lett. 12, 24. (1964).

18. M. Buhinstein, G.H. Strauss, and M. Bo Stearns, J. Appi. Phys. 37, 1334 (1966).

19. S. Hüfner and F. Matthias, Mösshaurr Spectroscopy and its Applications (IAEA, Vienna, 197?) p. 344.

20. S. A. Wender and N. Hershkowitz, Nucl. Instr. Methods 38, 105 (1972).

21. V. L. Gol 'danski 'i, E. F. Makarov, and V. V. Khrapov, Phys. Letters 3, 344 (1963); S. V. Karyagin, Dokl。

Akad. Nauk. SSSR 148, 1102 (1963)。

22. R. M. Housley, $V_{0}$ Gonser and R. W. Grant, Phys, Kev。 Letters 20, 1279 (1968).

23. Ho Do Pfannes and U. Gonser, Appl. Phys. I, 93 (1973).

24. C. Sauer, E. Matthias, and Ro L. Mỏssbauer, Phys。 Rev. Letters 21, 961 (1968).

25. F. E. Wagner, B. D. Dunlap, G. M. Kalvius, H. Schaller, R. Fel.scher, and Ii. Spieler, Phys. Rev. Letters 28, 530 (197?).

2f. W. Henninf, r. Baehre, and T. Kirnle, Thys. Isett. 3IB, 203 (1970).

27. G. Mo Kalvius, To E. Katila and O. V. Lounasmaa, Mössbauer Effect Metrodology, Vol. 5, Ed. I. J. Gruverman (Plenum Press, N.Y., 1970) p. 237.

28. G.K. Shenoy and H. Maletta (to be published). 
C QUADRUPCLE PATTERN FOR 5/2-7/2 TRANSITION IN 121 5B.

C GAUSS LEGENDRE INTEGRATION OF THE TRANSMISSION FUNCTION.... IMPLICIT REAL FS (A-H, O-Z)

IIMENSION DE (8EE). WTT (8EG), E (8EE). TRANS (BEE), XD(4E2), YCi $4 E 2$ ) DIMENSION VEL (B), CE(B).U(1EE). WT (1EO). B (1EE)

C NWT =NUMEER OF WEIGHTS. RQ=OUADRUPOLE MOMENT RATIO.

DATA PI. RQ. NWT /3.141592653589793.1.34.12/

C U\{1;...ULG; COORDINATES OF GAUSS LEGENGRE INTEGRATION.

C WTI1;...WTTLE) WEIGHTS FOR GAUSS LEGENDRE INTEGRATION.

DATA U(1),U(2),U(3)/E.9815606.0.9841173,0.7699627/

DATA U(4),U(5),U(6)/E.5873180.0.3678315,0.1253334/

DATA WT(1). WT (2). WT (3)/E.E471753.8.1869393.8.16E2783/

DATA WT(4). WT (5). WT (6:/E.2031674, 8.2334925,E.249147E/

DATA CQ/21..6..15...1.18..18..3..18./

C EQ =QUADRUPOLE INTERACTION. $S=$ ISOMER SHIFT. TA=ABSORBER THICKNESS.

$E Q=-2 \theta . E$

$S=0.0$

$T A=4.0$

C FSB = SOLRCE RESONANCE FRACTIONFSIGNAL/(SIGNAL + BACKEROUND) $F S B=1.0$

C WDS. WDA AND WDNAT ARE SOURCE. ABSGREER AND NATURAL WIDTHS.

WDS $=1$, ES

$W D A=1.05$

WDNAT $=1.05$

LINES $=8$

C VELIIS AND CGCI; GIVE THE LINE POSITION AND NORMALIZED INTENSITY OF

C I TH LINE IN THE HYPERF INE SFECTFUM.

$V E L(1)=3+E Q \div(R Q-1) / 4 .$.

$V E L(2 ;=5+E Q+(R Q / 7,-1) / 4.8$,

$V E L(3)=S+E G=\{R Q / 7,+0.23 / 4.0$

$V E L(4)=S+E Q=(R Q \div(-3 . E / 7 . E)-1) / 4 .$.

$V E L(5)=S+E Q=(R Q F(-3.0,7.0)+.2) / 4.0$

$V E L(6)=S+E O F(R O)(-3.0 / 7.0 j+0.8 j / 4.0$

$V E L(7)=S+E Q *(R Q \div(-5.17)+.B .2) / 4.0$ 


\section{APPENDIX A (cont ' $d$ )}

$V E L(B)=S+E Q F(R Q F(-5 . / 7)+.E .8) / 4 . E$

DO $2 I=1.8$

2 CEII $2 \times C E$ CI IFTAFWDNAT/(WDAF84.)

C VEXPT IS THE EXPERIMENTAL VELOCITY. VEXPT $=20.0$

C NP = NUMBER OF DATA POINTS.

$N P=1 \Xi 9$

$H N P=(N P / 2 . Q)+0.5$

C $\quad X D(I)=$ VELOCJTY OF I TH CHANNEL.

DO $5 I=1$. NP

$5 \times[(I)=V E X P T *(1-H N P) / H N P$

WDEXPT $=$ WDS + WDA

C ASSIENINE WEIGHTS TO THE NESATIVE COOPDINATES.

$M=N W T / 2$

DO $1 E I=1, M$

WT $(N W T-I+1) \times W T(I)$

$10 U(N W T-I+1)=-U(1)$

WDSSG $=$ (WUS/2, $) \neq \$ 2$

WDASQ $=(W D A / 2) \div$.

C ZONEWD = WIDTH OF THE INNER ZONE.

ZONEWD = VEXPT + S. \$WDEXPT

C NZONE = NUMBEF OF SUBZONES IN THE INNER REGION.

C NADSUE=NUMBER OF SUBZONES TO BE ALDED OR TO EE SUETRACTED FROM NZONE NZONE $=$ ZONEWD/WDEXPT

C NADSUB = PARAMETER TO INCREASE OR DECFEASE THE NUMBER OF SUEZONES. NADSUE $=$ E 


\section{APPENDIX A (cont' $d$ )}

NZONE = NZONE + NADSUE

C NUEER OF SUBZONES IN THE WING-NWING

NWING $=5$

C NZONET $=$ TOTAL NUMEER OF SUB ZONES IN ONE-HALF OF THE SPECTRUM. NZONET = NZONE + NWINE

C DEFINING THE GOUNDARIES OF THE SUBZONES IN THE WINGS. $B M I N=E . G$

DO $21 K=1$. NWINE

$21 B M I N=B M I N+10.5 K$

C $B(1)$ = VALUE OF THE NEGATIVE INFINITY FOR THE INTEGRATION.

$B(1)=-$ ZONEWD-EMINEWDEXPT

DO $15 I=2$. NWINE

$K=N W I N G+1-I$

$15 B(I+1)-B(I)+W D E X P T=10 . \neq 3 K$

C DEFINING THE BOUNDARIES OF THE INNER SUB ZONES.

C WDFACT = WIDTH OF INNER SUB ZONE.

WEF ACT = ZONEWD/NZONE

DO $16 \quad I=1$. NZONE

$K=N W I N G+I$

$16 \quad B(K+1 j=B(K)+H D F A C T$

C DEFINING SUE ZONE GOUNDARIES IN THE REMAINING HALF OF THE SPECTRUM. DO $17 I=1$. NZONET

$K=N Z O N E T+1-I$

$L=N Z O N E T+1+I$

1) $E(L)=-E(K)$

C CALCULATION OF GAUSS-LEGENDRE COORDINATES AND TRANS.

C NZONEZ TOTAL NUMBER OF SIIEZONES OVER ENTIRE SPECTRUM. NZONE2 = NZONET T2

NSTEP $=0$

DO $30 \mathrm{~L}=1$. NZONE2 


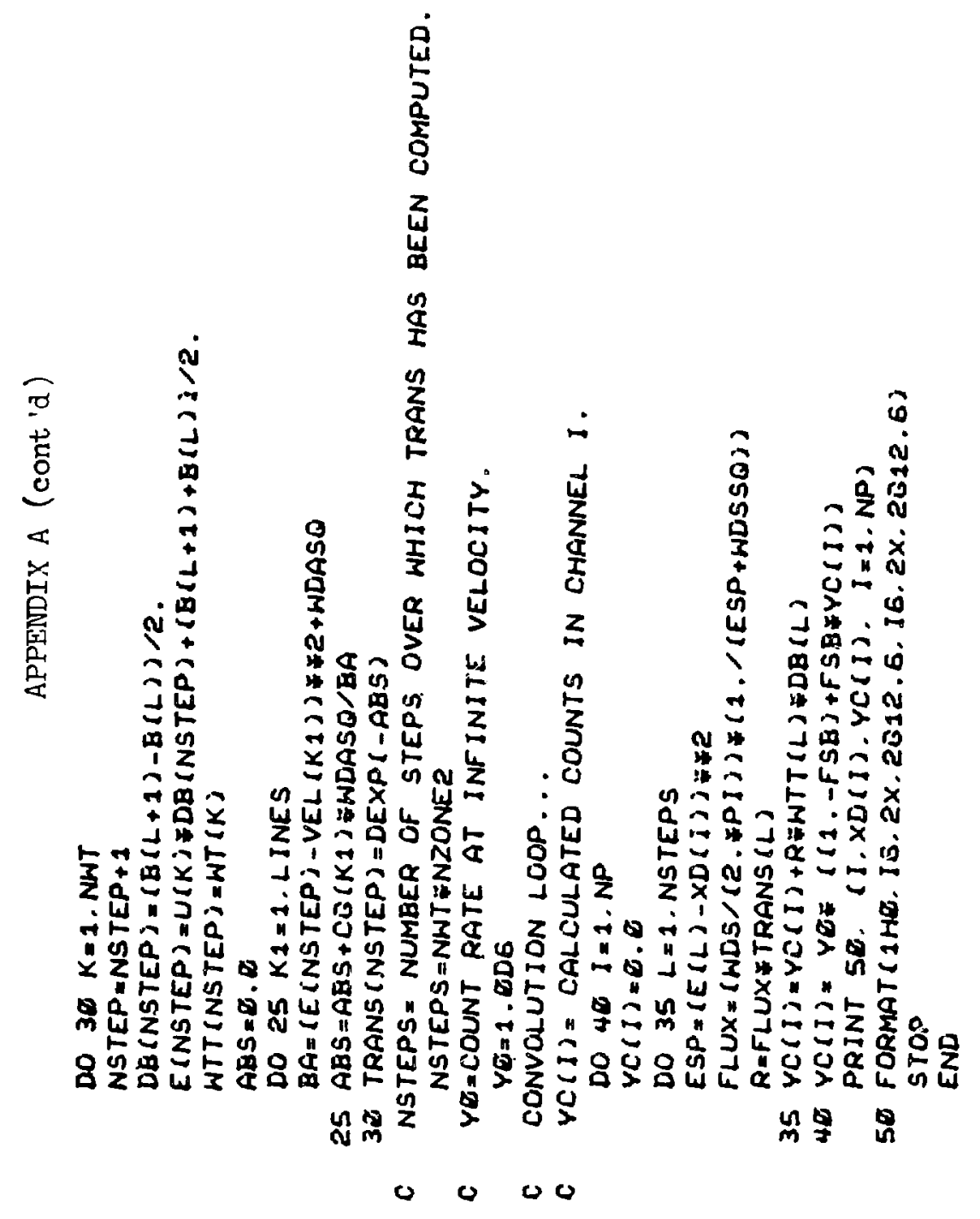


C CRANSHAN TRANSMISSION INTEGRAL FOR SINGLE SITE ANTIMONY. OUAD-

C RUPOLE ONLY: NP ODD OR EVEN. CONVO FLAE MUST BE ZERO OR ONE. O

C AND OP ARE CENTER POINTS IN TERMS LIKE $V=D V F(I-Q)$. N IS THE DUMAY

C ENERGY VARIAELE OF INTEGRATION: M REFERS TO DIFFERENT VELOCITIES

C

DIMENSION XD(4QE) . YD(4GQ) . YC (4EE)

DIMENGION F(482), X(20). VEL $(4,8), C E(8)$

DIMENSION SUMI (4OE). NING (4GO). SUM(4EB)

DIMENSION SUM(40OS). FLUXZP (BOE) . HING (4OO). SUMI (40E)

c

DATA CG/21..6..15..1..16..10..3..18./

CONVO = 1

$N P=199$

$F N P=N P$

NPHALF $=N P / 2$

$0=(N P / 2.0)+0.5$

$D O=(N P, 2 . Q)-N P / 2$

$N P E=2 F N P+2=D O$

$Q P=N P 2 / 2 . E+0.5$

c

$P I=D A R C O S(-2 . Q D E)$

DV $=0.2$

WDNAT $=1.85$

WDS $=1.05$

$F S B=2.0$

$R Q=2.34$

$Y O=1800000.6$

TA $=4.0$

$W D A=1.05$

$S=B .0$

$E O=-20.0$

$V E L(1)=5+(E Q / 4.0) 7(R Q-1.8)$ 


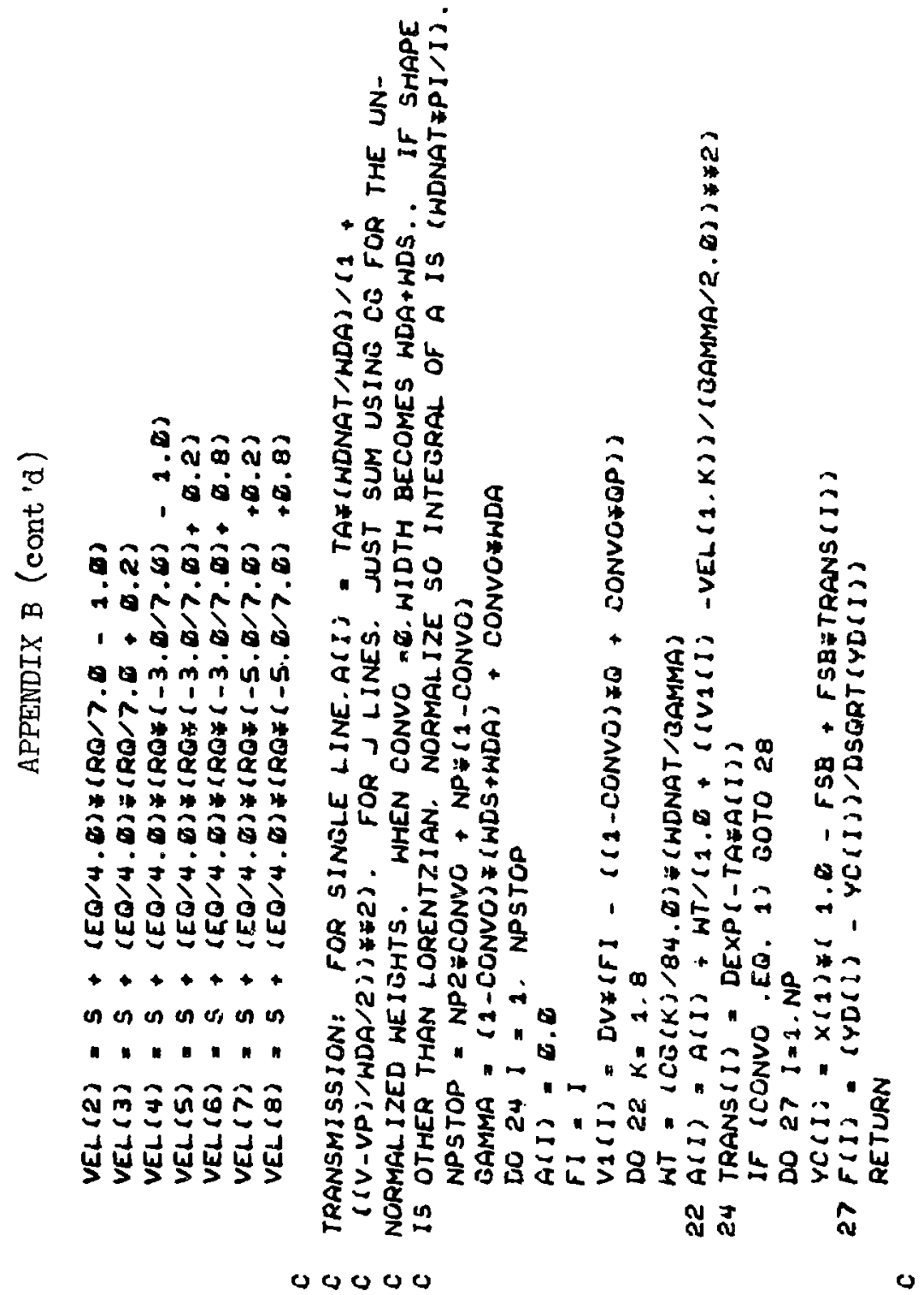




\section{APPENDIX B (cont'd )}

C CALCULATION OF FLUXZ

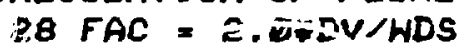

DO $3 B L=1$. NP2

$F L=L$

3E FLUXZ(L) $=(2 . E /(P I ¥ W D S)) /(1 . G+(F A C F(F L-F N P)) \neq \% 2)$

$c$

c CONVOLUTION CALCULATION

DO $50 M=1, N P$

SUM1 $(M)=0.0$

NSTART $=M-N P H A L F+1$

IF INSTART . LT . 1 INSTART $=1$

NSTOP $=M+N P 2-N P H A L F$

IF CNSTOP. GT . NPE) NSTOP =NPE

DELM = DATAN (FACF (NSTART -M-NPHALF - O.5-2.EFDQ))

DELP = DATAN(FAC $(N S T O P-M-N P H A L F+0.5-2.0 * D Q))$

WING $(M)=(1 . E / P I)=(D E L M-D E L P)+1.0$

LO $4 E N=N S T A R T$. NSTOP

4E $\operatorname{SUM} 2(M)=\operatorname{SUM} 2(M)+\operatorname{TRANS}(N) * F L U \times Z(N+N P H A L F-M)$

$\operatorname{SUM}(M)=\operatorname{DV} F \operatorname{SUM} 2(M)+W I N G(M)$

YC(J) = YG:(1.E-FSBF(1.E-SUMLJ)

$50 F(J)=(Y D(J)-Y C(j)) / D S O R T(Y D(J))$

RETURN

c

15 PRINT 940. (I. XD(I), YC(I) I I $1 . N P$ )

94E FORMAT (1HE. I6. $2 \times .2612 .6,16.2612 .6$ )

RETURN

END 